\title{
Hepatoprotective Effects of Chai-Hu-Ching- Kan-Tang on Acetaminophen-Induced Acute Liver Injury in Rats
}

\author{
Tzu-Hsiang Lin, ${ }^{*}$ Lean-Teik Ng, ${ }^{\S}$ Feng-Lin Yen ${ }^{\dagger}$ and Chun-Ching Lin ${ }^{\ddagger}$ \\ ${ }^{*}$ Graduate Institute of Natural Products, ${ }^{\dagger}$ Graduate Institute of Pharmaceutical Sciences \\ ${ }^{\ddagger}$ Faculty of Pharmacy, College of Pharmacy \\ Kaohsiung Medical University, Kaohsiung, Taiwan \\ ${ }^{\S}$ Department of Biotechnology, Tajen University, Pingtung, Taiwan
}

\begin{abstract}
Chai-Hu-Ching-Kan-Tang (CHCKT) is one of the traditional Chinese medicine prescriptions commonly used to treat liver diseases. In this study, we evaluated the hepatoprotective effects of aqueous CHCKT extract at various concentrations $(125,250$ and $500 \mathrm{mg} / \mathrm{kg}$ body weight) on acetaminophen (APAP)-induced acute liver injury in rats. Results showed that CHCKT treatments significantly decreased the level of serum glutamic oxaloacetic transaminase (sGOT) and glutamic pyruvic transaminase (sGPT) in APAP-treated groups. CHCKT also significantly decreased the level of lipid peroxides and increased the activity of antioxidant enzymes (i.e. SOD and GPx). Histopathological observation further confirmed the hepatoprotective activity of CHCKT as indicated by the amelioration in the central necrosis and fatty changes of the liver after APAP induction. Interestingly, the hepatoprotective activity of CHCKT at concentrations $125 \sim 500 \mathrm{mg} / \mathrm{kg}$ appeared to be as good as $12.5 \mathrm{mg} / \mathrm{kg}$ silymarin (a commercial hepatoprotective agent). Taken together, these results suggest that aqueous extract of CHCKT possesses potent hepatoprotective effects agianst APAP-induced liver injury in rats.
\end{abstract}

Keywords: Hepatoprotective; Antioxidant; Acetaminophen; Chai-Hu-Ching-Kan-Tang.

\section{Introduction}

Liver injury can be caused by different agents, including chemicals, alcohol, virus and auto-immune diseases (Sugiyama et al., 1999). Acetaminophen (APAP) is a widely used analgesic and antipyretic drug in many countries. When taken in overdose, it may cause

Correspondence to: Professor Chun-Ching Lin, Faculty of Pharmacy, College of Pharmacy, Kaohsiung Medical University, 100 Shih-Chuan 1st Road, Kaohsiung 807, Taiwan, R.O.C. Tel: (+886) 7-312-1101 (ext. 2122), Fax: (+886) 7-313-5215, E-mail: aalin@kmu.edu.tw 
centrilobular hepatic necrosis (Mitchell et al., 1973). The hepatotoxicity induced by APAP is thought to be caused by N-acetyl-para-benzoquinoneimine (NAPQI), a cytochrome P-450-mediated intermediate metabolite (Dahlin et al., 1984). This metabolite is normally detoxified by conjugation with glutathione (Coles et al., 1988). When covalently binding to cellular macromolecules, it can lead to the production of reactive oxygen species (ROS) (Lores Arnaiz et al., 1995; Rogers et al., 1997). An imbalance between the formation and removal of these ROS may lead to the development of oxidative stress. This phenomenon is recognized to play an important role in drug toxicity (Andrade et al., 1998). The major defense mechanism in a living body is the antioxidant enzymes, namely glutathione peroxidase (GPx), superoxide dismutase (SOD) and catalase (CAT). These enzymes convert active oxygen molecules into non-toxic compounds (Halliwell and Gutteridge, 1984; Hochstein and Atallah, 1988) and consequently protect the liver against the ROS damage.

Compared to Xiao Chai Hu Tang (XCHT), Chai-Hu-Ching-Kan-Tang (CHCKT) is a less known prescription in traditional Chinese medicine for treating liver diseases. The main ingredient of these two prescriptions is Bupleurum radix. Studies have shown that XCHT possessed antioxidant (Sakaguchi et al., 1993; Egashira et al., 1999), immunomodulating (Fujiwara et al., 1995; Yamashiki et al., 1997) and antiproliferative (Kayano et al., 1998; Liu et al., 1998) activities. It ameliorates chronic viral infected liver diseases (Kayano et al., 1998; Liu et al., 1998), enhances production of IFN (Matsuura et al., 1993; Huang et al., 2001) and antibodies in patients with chronic hepatitis (Kakumu et al., 1991). However, it is unknown whether CHCKT possesses similar pharmacological properties. In this study, our aim was to investigate the hepatoprotective effects of CHCKT against APAP-induced liver injury in rats.

\section{Materials and Methods}

\section{Chemicals}

Thiobarbituric acid (TBA), nitrobluetetrazolium (NBT), 1,1,3,3-tetraethoxypropane (TEP), 5,5'-dithiobis-p-nitrobenzoic acid (DTNB), reduced glutathione (GSH) and catalase were purchased from Sigma Chemicals Co. (St. Louis, MO, USA). All other chemicals and reagent used were in analytical grade.

\section{Preparation of Chai-Hu-Ching-Kan-Tang (CHCKT) Extract}

The formulation of CHCKT is consisted of eight different medical plant ingredients as shown in Table 1. They were purchased from an herbal grocery in Kaohsiung city, Taiwan. The authenticity of these ingredients was confirmed by Dr. M.H. Yen (Graduate Institute of Natural Products, Kaohsiung Medical University, Taiwan) using histological technique.

To prepare the aqueous extract of CHCKT, $100 \mathrm{~g}$ of dried materials was extracted with 1 liter of boiling water for 2 hours. After the supernatant of the first extraction was removed, an additional 1 liter of distilled water was added and the sample was further subjected 
Table 1. Composition of Chai-Hu-Ching-Kan-Tang (CHCKT)

\begin{tabular}{lc}
\hline Ingredients & Weight $(\mathrm{g})$ \\
\hline Bupleurum kaoi & 4.0 \\
Scutellaria baicalensis & 3.0 \\
Panax ginseng & 3.0 \\
Ligusticum chuaxing & 3.0 \\
Gardenia jasminoides & 3.0 \\
Forsythia suspensa & 2.0 \\
Platycodon grandiflorum & 2.0 \\
Glycyrrhizae uralensis & 1.0 \\
\hline
\end{tabular}

to another 2 hours of extraction. The same procedure was repeated for three times. The decoction obtained from the three extractions was combined, filtered, concentrated and lyophilized. The yield of dried extract was about $38 \%$, which was collected and stored at $-21^{\circ} \mathrm{C}$ until use.

\section{Animals}

Male Wistar rats, at about 6 weeks of age (220-250 g), were purchased from the National Laboratory of Animal Breeding and Research Center (Taipei, Taiwan). They were housed in a controlled environment with temperature maintained at $22 \pm 3^{\circ} \mathrm{C}$ and humidity at $55 \pm$ $5 \%$ under a 12:12 hours light/dark cycle. Animals were fed a standard laboratory diet and tap water ad libitum. After a week of adaptation, animals were subjected to various treatments for hepatotoxicity studies. All experimental protocols were performed according to the Guide for the Care and Use of Laboratory Animals (National Institutes of Health, publication No. 86-23, revised 1985).

\section{Experimental Design}

The experiment was conducted according to the modified procedures as described previously (Lin et al., 1995). Rats were randomly divided into six groups of eight. Group 1 served as a normal control, which received orally $10 \mathrm{ml} / \mathrm{kg}$ of normal saline $(0.9 \% \mathrm{NaCl})$. Group 2 was treated with an oral dose of water $(5 \mathrm{ml} / \mathrm{kg})$ twice a day for one week before APAP treatment. Group 3 and Groups 4 6 were treated in a similar fashion as Group 2, except that the reference drug silymarin $(12.5 \mathrm{mg} / \mathrm{kg}$; suspended in $1 \% \mathrm{CMC})$ and CHCKT extracts $(125,250$ and $500 \mathrm{mg} / \mathrm{kg})$ were given instead of the saline, respectively. On day 8 , animals from groups 2 6 were intraperitoneally (i.p.) administered with a single dose of APAP (750 mg/kg; in 25\% PEG400 solution). On the following day, all rats were sacrificed under ether anesthesia. Blood was obtained by cardiac puncture, and liver samples were collected for biochemical assays. A portion of the liver was fixed in $10 \%$ formalin for histopathological studies. 
Biological Assays

Collected blood samples were centrifuged at $3000 \times \mathrm{g}$ at $4{ }^{\circ} \mathrm{C}$ for $10 \mathrm{~min}$ to obtain the serum, which was then subjected to sGOT and sGPT analysis according to procedures described by manufacturer using ELISA kits (Beckman Inc., Fullerton, CA, USA).

Liver tissues were homogenized in four volumes of ice-cold $20 \mathrm{mM}$ Tris- $\mathrm{HCl}$ (pH 7.4) containing $0.15 \mathrm{M} \mathrm{KCl}$ using Potter-Elvehjem homogenizer with a Teflon pestle. The homogenates were centrifuged at $3200 \times \mathrm{g}$ for $20 \mathrm{~min}$ at $4^{\circ} \mathrm{C}$ and the supernatant was subjected to various biochemical analyses. Catalase (CAT) activity was determined according to the methods of Aebi (1984) and, Beers and Sizer (1952). Superoxide dismutase (SOD) activity was determined by the method of Beauchamp and Fridovich (1971). Total glutathione peroxidase (GPx) was done with method of Flohe and Gunzler (1984). Lipid peroxidation in liver homogenates was ascertained by the formation of malondialdehyde (MDA) and measured by the thiobarbituric acid reactive method according to Ohkawa et al. (1979). The protein concentration was measured by the method of Lowry et al. (1951).

\section{Histopathological Observation}

The APAP-induced liver necrosis was evaluated using hematoxylin and eosin ( $\mathrm{H} \& \mathrm{E})$ staining. In brief, immediately after collecting the blood under ether anaesthesia, the rat's liver was removed and fixed in $10 \%$ formalin solution for a week. The liver tissue was dehydrated with a sequence of ethanol solutions, embedded in paraffin and sectioned at thickness of $5 \mu \mathrm{m}$ followed by staining with haematoxylin-eosin dye before subjecting to photomicroscopic observation.

\section{Statistical Analysis}

All experimental data were expressed as means \pm standard deviations (SD). Statistical analysis was performed by one way analysis of variance (ANOVA), followed by Scheffe's multiple range test. $\mathrm{p}<0.05$ was considered as significance.

\section{Results}

Effects of CHCKT on Serum Glutamic Oxaloacetic Transaminase (sGOT) and Glutamic Pyruvic Transaminase (sGPT)

Results showed that a dose of $750 \mathrm{mg} / \mathrm{kg}$ APAP significantly caused the liver injury in rats at 24 hours after injection, as indicated by the substantial increase of the sGOT and sGPT activities (Table 2). Administration of CHCKT at 125, 250 and $500 \mathrm{mg} / \mathrm{kg}$ to rats significantly decreased the sGOT and sGPT levels in a dose-dependent manner. Interestingly, the decrease of sGOT and sGPT activities after CHCKT treatment was as effective as $12.5 \mathrm{mg} / \mathrm{kg}$ silymarin. 
Table 2. Effect of CHCKT on the Levels of Serum Glutamic Oxaloacetic Transaminase (sGOT) and Serum Glutamic Pyruvic Transaminase (sGPT) in APAP-Induced Rats

\begin{tabular}{lcc}
\hline Treatments & sGOT (IU/L) & sGPT (IU/L) \\
\hline Control & $291.0 \pm 44.6^{\mathrm{d}}$ & $83.75 \pm 8.1^{\mathrm{c}}$ \\
APAP & $3006.1 \pm 1478.9^{\mathrm{a}}$ & $1560.2 \pm 763.1^{\mathrm{a}}$ \\
APAP + Silymarin $12.5 \mathrm{mg} / \mathrm{kg}$ & $537.3 \pm 134.6^{\mathrm{b}}$ & $136.3 \pm 32.6^{\mathrm{b}}$ \\
APAP + CHCKT $125 \mathrm{mg} / \mathrm{kg}$ & $427.2 \pm 77.5^{\mathrm{bc}}$ & $132.8 \pm 37.5^{\mathrm{b}}$ \\
APAP + CHCKT $250 \mathrm{mg} / \mathrm{kg}$ & $354.0 \pm 55.1^{\mathrm{bcd}}$ & $108.0 \pm 30.9^{\mathrm{bc}}$ \\
APAP + CHCKT $500 \mathrm{mg} / \mathrm{kg}$ & $402.2 \pm 2.2^{\mathrm{bc}}$ & $168.0 \pm 41.3^{\mathrm{b}}$ \\
\hline
\end{tabular}

Data were presented as means \pm SD from eight independent analyses. Values within the same column with the different superscript letters were significantly different at $\mathrm{p}<0.05$ as analyzed by Scheffe's multiple range tests.

Table 3. Biochemical Assessment of APAP-Induced Liver Injury

\begin{tabular}{lcccc}
\hline \multirow{2}{*}{ Treatments } & \multicolumn{2}{c}{ U/mg Protein } & \multicolumn{2}{c}{ nmol/mg Protein } \\
\cline { 2 - 5 } & SOD & GSH-Px & CAT & MDA \\
\hline Control & $2.99 \pm 0.08^{\mathrm{a}}$ & $17.88 \pm 1.1^{\mathrm{a}}$ & $80.56 \pm 10.32^{\mathrm{a}}$ & $1.45 \pm 0.36^{\mathrm{b}}$ \\
APAP & $2.23 \pm 0.34^{\mathrm{b}}$ & $14.83 \pm 0.85^{\mathrm{b}}$ & $52.38 \pm 10.57^{\mathrm{b}}$ & $2.94 \pm 0.52^{\mathrm{a}}$ \\
APAP + Silymarin $12.5 \mathrm{mg} / \mathrm{kg}$ & $3.28 \pm 0.27^{\mathrm{a}}$ & $17.88 \pm 1.09^{\mathrm{a}}$ & $31.83 \pm 12.07^{\mathrm{c}}$ & $1.13 \pm 0.44^{\mathrm{b}}$ \\
APAP + CHCKT 125mg/kg & $4.01 \pm 0.34^{\mathrm{a}}$ & $18.31 \pm 1.74^{\mathrm{a}}$ & $60.68 \pm 6.84^{\mathrm{b}}$ & $1.64 \pm 0.31^{\mathrm{b}}$ \\
APAP + CHCKT 250mg/kg & $3.75 \pm 0.38^{\mathrm{a}}$ & $17.95 \pm 1.48^{\mathrm{a}}$ & $59.70 \pm 9.85^{\mathrm{b}}$ & $1.07 \pm 0.22^{\mathrm{b}}$ \\
APAP + CHCKT 500mg/kg & $3.48 \pm 0.23^{\mathrm{a}}$ & $20.75 \pm 0.71^{\mathrm{a}}$ & $61.58 \pm 10.93^{\mathrm{b}}$ & $0.84 \pm 0.27^{\mathrm{c}}$ \\
\hline
\end{tabular}

Data were presented as means \pm SD from eight independent analyses. Values within the same column with the different superscript letters were significantly different at $\mathrm{p}<0.05$ as analyzed by Scheffe's multiple range tests. APAP: $750 \mathrm{mg} / \mathrm{kg}$, SOD: superoxide dismutase (U/mg protein), GPx: glutathione peroxidase (U/mg protein), CAT: catalase (U/mg protein), MDA: malondialdehyde (nmole/mg protein).

\section{Effects of CHCKT on Liver Antioxidant Enzymes}

Table 2 showed the results of SOD, GPx and CAT concentrations in rat liver after challenged by APAP. Compared to the normal control group, APAP treatment caused a significant decrease in GPx and SOD activities. However, pre-treatment of rats with CHCKT at concentrations $125 \sim 500 \mathrm{mg} / \mathrm{kg}$ significantly increased the levels of SOD and GPx, and these levels were not different from those of the control and silymarin treated groups. APAP treatment or together with CHCKT resulted in a significant reduction of CAT level as compared to the control group. It was a surprise to note that co-treatment of APAP and silymarin caused a dramatic decrease of CAT activity.

\section{Effects of CHCKT on Hepatic Lipid Peroxidation}

Compared to the untreated control group, APAP treatment significantly increased the concentration of MDA in rat liver by two fold (Table 3). However, when rats were administered with CHCKT, the liver MDA concentration was found to return to the level 
of control group $(1.45 \pm 0.36 \mathrm{nmol} / \mathrm{g})$ and were significantly different from the APAP treated group $(2.94 \pm 0.52 \mathrm{nmol} / \mathrm{g})$. The level of MDA concentration in rats treated with CHCKT at $125 \sim 500 \mathrm{mg} / \mathrm{kg}$ was not significantly different from that of the control and silymarin treated groups.

\section{Histopathological Observation}

Results of the histopathological observation on the liver tissues after various treatments are shown in Fig. 1. Figure 1A shows a representative photomicrograph of a liver obtained from a normal control rat, where there was no necrosis, inflammation nor vascular degeneration. However, in rat administered with APAP, a patch-like necrotic and hemorrhagic necrosis was noted in the central and middle zones, with many pyknotic cells around the lesions (Fig. 1B). However, treatment with CHCKT significantly reversed, to a large extent, the hepatic lesions produced by APAP (Figs. 1D, 1E and 1F). In animals treated with $125 \sim 500 \mathrm{mg} / \mathrm{kg}$ CHCKT, the changes of morphological appearance of liver was similar to that of $12.5 \mathrm{mg} / \mathrm{kg}$ silymarin.

\section{Discussion}

The present study showed that CHCKT possesses potent hepatoprotective activity as demonstrated by a significant decrease in sGOT and sGPT concentrations and improvement in liver morphological appearance. This observation was further supported by the increase of activities of antioxidant enzymes and the decrease of the concentration of lipid peroxides.

APAP is well-known to be metabolized in the liver. Its mechanism involved in the development of hepatoxicity is well documented (Goldin et al., 1996; Hinson et al., 2002). APAP metabolized to the reactive metabolite NAPQI and its detoxification by glutathione is a critical determinant in APAP toxicity (Mitchell et al., 1973; Nelson, 1990). NAPQI, a toxic electrophile, binds convalently to tissue macromolecules (Mitchell et al., 1973) and alters the homeostasis of calcium (Moore et al., 1985). It is a very active free radical which can readily oxidize lipid and thiol (-SH) groups, as well as changing the stability of calcium ion. Consequently, it triggers the process of lipid peroxidation to cause the damage in liver cells (Moldeus, 1978; Moore et al., 1985). Hepatic necrosis occurs only when the amount of reactive NAPQI produced exceeds the binding capacity of glutathione. In this study, APAP at $750 \mathrm{mg} / \mathrm{kg}$ produced severe centrizonal hemorrhagic hepatic necrosis, ballooning degeneration, and fatty changes. It also remarkably increased the levels of sGOT and sGPT.

Serum transaminase elevation has been reported to be associated with a number of inflammatory disorders and hepatocellular damage (Sihna et al., 1972). Leakage of large quantities of enzymes into the blood stream is often associated with massive necrosis of the liver (Rees and Specter, 1961). A single injection of APAP to rat is widely used as a model for investigating the hepatoprotective effects of natural products and crude drugs. In this study, the elevation of serum transaminases (i.e. sGOT and sGPT) level reflected 

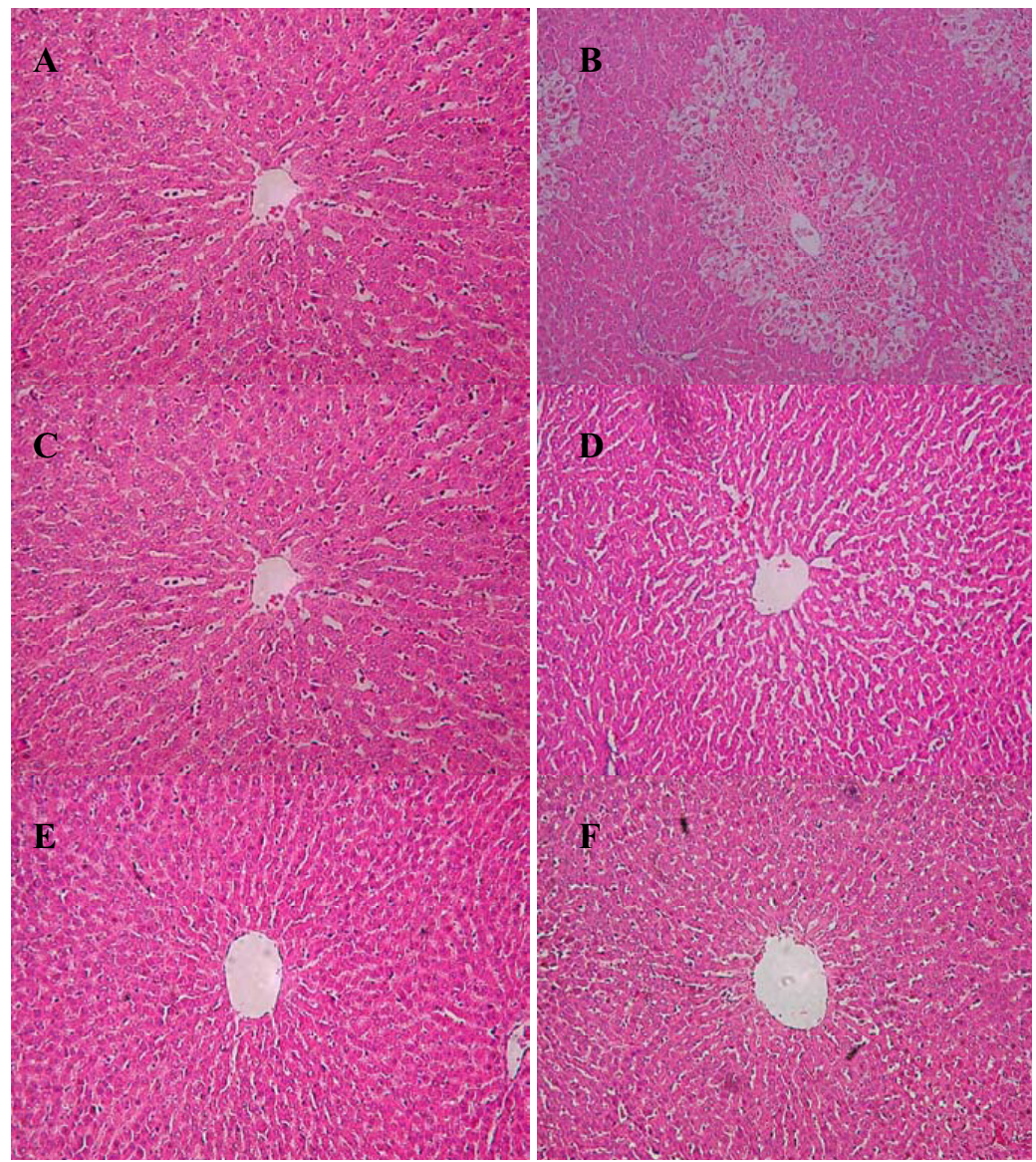

Figure 1. The photomicrographs $(\times 200)$ of liver section in APAP and CHCKT treated rats. Liver sections were stained with haematoxylin and eosin (HE stain $176 \mathrm{x}$ ). A: normal; B: APAP-induced; C: APAP+Silymarin; D: APAP+CHCKT 125mg/kg; E: APAP+CHCKT 250mg/kg; F: APAP+CHCKT 500mg/kg.

in the APAP-treated animals has indicated the occurrence of hepatocellular damage. The levels of these transaminases were significantly reversed by CHCKT treatment at doses 125,250 and $500 \mathrm{mg} / \mathrm{kg}$. These results suggest that CHCKT may improve a generally damaged condition and suppress enzyme leakage from cellular membranes. Interestingly, the protective effect of CHCKT at all tested concentrations was found to be as good as $12.5 \mathrm{mg} / \mathrm{kg}$ silymarin (a commercial hepatoprotective agent).

Previous studies suggest that an increase in concentration of superoxide is associated with APAP (James et al., 2003). Mitochondrial dysfunction may be an important mechanism in APAP-induced hepatotoxicity. It is known that mitochondrial permeability transition occurs with the formation of superoxide, and this may be the source of superoxide that leads to peroxynitrite and tyrosine nitration. Studies with encapsulated SOD administration showed a decrease in the APAP-induced toxicity in rats. This result was postulated to be 
the superoxide scavenging activity of SOD (Nakae et al., 1990). We showed that SOD activity was significantly increased in rats after CHCKT treatment, suggesting that the hepatoprotective effect of CHCKT could be due to its antioxidant properties.

GPx plays an important role in the stabilization of polyunsaturated membrane lipids (Christophersen, 1968). Under conditions of NAPQI formation following toxic APAP doses, glutathione concentrations may be very low in the centrilobular cells. GPx, the major peroxide detoxification enzyme, may functions inefficiently under conditions of glutathione depletion (Zakowski and Tappel, 1978). The detoxification of hydrogen peroxide is primarily through the activity of glutathione, which is essential in preventing hydroxyl radical formation (Hochstein and Atallah, 1988). The present study showed that activity of GPx was significantly increased in rats after treating with CHCKT. However, there was no difference in the CAT activity between APAP and APAP plus CHCKT treatments. These results suggest that CHCKT may detoxify the hydrogen peroxide through action of GPX and GSH, but not of CAT.

The MDA concentration increased significantly following APAP-induced hepatotoxicity as compared to the untreated control group, suggesting that an obvious lipid peroxidation occurred in the liver. CHCKT effectively inhibited the lipid peroxidation as demonstrated by the returning of MDA concentration to the level of the untreated control group. The hepatoprotective effect of CHCKT may be due to the inhibition of NAPQI formation or radical peroxidation of membrane lipids.

The histological changes associated with the hepatoprotective activity of CHCKT basically support the estimation of the serum enzymes. Administration of APAP to an animal leads to centrilobular necrosis in the liver, including infiltration of lymphocyte, Kupffer cell, fatty change and ballooning degeneration. The inflammation of hepatocyte and ballooning degeneration was reversed after administration of CHCKT. These results suggest that the inhibition of serum transaminases elevation and hepatic damage may play an important role in the protective effect of CHCKT on APAP-induced hepatocellular destruction.

Detailed chemical studies on the ingredients of CHCKT showed that these herbal materials contain a number of bioactive compounds. These compounds have various pharmacological actions, and are assumed to be responsible, at least part, for the pharmacological effects of CHCKT. For example, glycyrrhizin and glycyrrhizic acid, bioactive components of Glycyrrhizae radix, showed hepatoprotective (Taira et al., 2004), anti-inflammatory (Francischetti et al., 1997; Kroes et al., 1997), anti-ulcer (Zani et al., 1993), and anti-HIV (Ito et al., 1987) activities. Baicalin and baicalein of Scutellaria radix exhibited anti-inflammatory (Lin and Shieh, 1996; Taira et al., 2004), anti-cancer (Ikemoto et al., 2000) and anti-HIV (Ikemoto et al., 2000) activities, as well as stimulating IL-1 $\beta$ production (Kimura et al., 1997). Ginsenoside Rb1 and ginsenoside Rg1, active components of Panax ginseng showed hepatoprotective (Lee et al., 2005), neuroprotection (Cheng et al., 2005; Lee et al., 2005), anti-amnestic (Cheng et al., 2005), anti-aging (Cheng et al., 2005) and immunomodulatory (Rivera et al., 2005) activities.

In conclusion, CHCKT showed excellent protection against APAP-induced liver damage in rats. In spite of the unknown mechanism(s) of action, the enhanced levels of 
antioxidative enzymes and diminished lipid peroxides level in rat liver suggested that the mechanism(s) involved in hepatoprotection of liver could be derived from the antioxidant activities of CHCKT. This observation was further supported by the improvement of liver morphological appearance after CHCKT administration. Taken together, the present study has shown the potential of CHCKT against APAP-induced hepatotoxicity. Hence, the exact hepatoprotective mechanism of CHCKT and the development of its crude extract as an effective remedy for hepatic injuries warrant further study.

\section{References}

Aebi, H. Catalase in vitro. Methods Enzymol. 105: 121-126, 1984.

Andrade, F.H., M.B. Reid, D.G. Allen and H. Westerblad. Effect of hydrogen peroxide and dithiothreitol on contractile function of single skeletal muscle fibres from the mouse. J. Physiol. 509(Pt 2): 565-575, 1998.

Beauchamp, C. and I. Fridovich. Superoxide dismutase: improved assays and an assay applicable to acrylamide gels. Anal. Biochem. 44: 276-287, 1971.

Beers, Jr. R.F. and I.W. Sizer. A spectrophotometric method for measuring the breakdown of hydrogen peroxide by catalase. J. Biol. Chem. 195: 133-140, 1952.

Cheng, Y., L.H. Shen and J.T. Zhang. Anti-amnestic and anti-aging effects of ginsenoside Rg1 and Rb1 and its mechanism of action. Acta Pharmacol. Sin. 26: 143-149, 2005.

Christophersen, B.O. Formation of monohydroxy-polyenic fatty acids from lipid peroxides by a glutathione peroxidase. Biochim. Biophys. Acta 164: 35-46, 1968.

Coles, B., I. Wilson, P. Wardman, J.A. Hinson, S.D. Nelson and B. Ketterer. The spontaneous and enzymatic reaction of $\mathrm{N}$-acetyl-p-benzoquinonimine with glutathione: a stopped-flow kinetic study. Arch. Biochem. Biophys. 264: 253-260, 1988.

Dahlin, D.C., G.T. Miwa, A.Y. Lu and S.D. Nelson. N-acetyl-p-benzoquinone imine: a cytochrome P-450-mediated oxidation product of acetaminophen. Proc. Natl. Acad. Sci. USA 81: 1327-1331, 1984.

Egashira, T., F. Takayama, Y. Yamanaka and Y. Komatsu. Monitoring of radical scavenging activity of peroral administration of the Kampo medicine sho-saiko-to in rats. Jpn. J. Pharmacol. 80: 379-382, 1999.

Francischetti, I.M., R.Q. Monteiro and J.A. Guimaraes. Identification of glycyrrhizin as a thrombin inhibitor. Biochem. Biophys. Res. Commun. 235: 259-263, 1997.

Fujiwara, K., S. Mochida, S. Nagoshi, O. Iijima, Y. Matsuzaki, S. Takeda and M. Aburada. Regulation of hepatic macrophage function by oral administration of xiao-chai-hu-tang (sho-saiko-to, TJ-9) in rats. J. Ethnopharmacol. 46: 107-114, 1995.

Flohe, L., and W.A. Gunzler. Assays of glutathione peroxidase. Methods Enzymol. 105: 114-121, 1984.

Goldin, R.D., I.D. Ratnayaka, C.S. Breach, I.N. Brown and S.N. Wichramasingfe. Role of macrophages in acetaminophen (paracetamol)-induced hepatotoxicity. J. Pathol. 179: 432-435, 1996.

Halliwell, B. and J.M. Gutteridge. Lipid peroxidation, oxygen radicals, cell damage, and antioxidant therapy. Lancet 1: 1396-1397, 1984.

Hinson, J.A., A.R. Bucci, L.K. Irwin, S.L. Michael and P.R. Mayeux. Effect of inhibitors of nitric oxide synthase on acetaminophen-induced hepatotoxicity in mice. Nitric Oxide 6: 160-167, 2002. 
Hochstein, P., and A.S. Atallah. The nature of oxidants and antioxidant systems in the inhibition of mutation and cancer. Mutat. Res. 202: 363-375, 1988.

Huang, X.X., M. Yamashiki, K. Nakatani, T. Nobori and A. Mase. Semi-quantitative analysis of cytokine mRNA expression induced by the herbal medicine sho-saiko-to (TJ-9) using a Gel Doc system. J. Clin. Lab. Anal. 15: 199-209, 2001.

Ikemoto, S., K. Sugimura, N. Yoshida, R. Yasumoto, S. Wada, K. Yamamoto and T. Kishimoto. Antitumor effects of Scutellariae radix and its components baicalein, baicalin, and wogonin on bladder cancer cell lines. Urology 55: 951-955, 2000.

Ito, M., H. Nakashima, M. Baba, R. Pauwels, E. De Clercq, S. Shigeta and N. Yamamoto. Inhibitory effect of glycyrrhizin on the in vitro infectivity and cytopathic activity of the human immunodeficiency virus [HIV (HTLV-III/LAV)]. Antiviral Res. 7: 127-137, 1987.

James, L.P., P.R. Mayeux and J.A. Hinson. Acetaminophen-induced hepatotoxicity. Drug Metab. Dispos. 31: 1499-1506, 2003.

Kakumu, S., K. Yoshioka, T. Wakita and T. Ishikawa. Effects of TJ-9 sho-saiko-to (kampo medicine) on interferon gamma and antibody production specific for hepatitis B virus antigen in patients with type B chronic hepatitis. Int. J. Immunopharmacol. 13: 141-146, 1991.

Kayano, K., I. Sakaida, K. Uchida and K. Okita. Inhibitory effects of the herbal medicine sho-saikoto (TJ-9) on cell proliferation and procollagen gene expressions in cultured rat hepatic stellate cells. J. Hepatol. 29: 642-649, 1998.

Kimura, Y., N. Matsushita, and H. Okuda. Effects of baicalein isolated from Scutellaria baicalensis on interleukin 1 beta- and tumor necrosis factor alpha-induced adhesion molecule expression in cultured human umbilical vein endothelial cells. J. Ethnopharmacol. 57: 63-67, 1997.

Kroes, B.H., C.J. Beukelman, A.J. van den Berg, G.J. Wolbink, H. van Dijk and R.P. Labadie. Inhibition of human complement by beta-glycyrrhetinic acid. Immunology 90: 115-120, 1997.

Lee, H.U., E.A. Bae, M.J. Han, N.J. Kim and D.H. Kim. Hepatoprotective effect of ginsenoside Rb1 and compound K on tert-butyl hydroperoxide-induced liver injury. Liver Int. 25: 1069-1073, 2005.

Lin, C.C. and D.E. Shieh. The anti-inflammatory activity of Scutellaria rivularis extracts and its active components, baicalin, baicalein and wogonin. Am. J. Chin. Med. 24: 31-36, 1996.

Lin, C.C., C.C. Tsai and M.H. Yen. The evaluation of hepatoprotective effects of Taiwan folk medicine 'Teng-Khia-U'. J. Ethnopharmacol. 45: 133-123, 1995.

Liu, W., M. Kato, A.A. Akhand, A. Hayakawa, M. Takemura, S. Yoshida, H. Suzuki and I. Nakashima. The herbal medicine sho-saiko-to inhibits the growth of malignant melanoma cells by upregulating Fas-mediated apoptosis and arresting cell cycle through downregulation of cyclin dependent kinases. Int. J. Oncol. 12: 1321-1326, 1998.

Lores Arnaiz, S., S. Llesuy, J.C. Cutrin and A. Boveris. Oxidative stress by acute acetaminophen administration in mouse liver. Free Radic. Biol. Med. 19: 303-310, 1995.

Lowry, O.H., N.J. Rosebrough, A.L. Farr and R.J. Randall. Protein measurement with the folin phenol reagent. J. Biol. Chem. 193: 265-275, 1951.

Matsuura, K., T. Kawakita, S. Nakai, Y. Saito, A. Suzuki and K. Nomoto. Role of B-lymphocytes in the immunopharmacological effects of a traditional Chinese medicine, xiao-chai-hu-tang (shosaiko-to). Int. J. Immunopharmacol. 15: 237-243, 1993.

Mitchell, J.R., D.J. Jollow, W.Z. Potter, D.C. Davis, J.R. Gillette and B.B. Brodie. Acetaminopheninduced hepatic necrosis. I. Role of drug metabolism. J. Pharmacol. Exp. Ther. 187: 185-194, 1973. 
Moldeus, P. Paracetamol metabolism and toxicity in isolated hepatocytes from rat and mouse. Biochem. Pharmacol. 27: 2859-2863, 1978.

Moore, M., H. Thor, G. Moore, S. Nelson, P. Moldeus and S. Orrenius. The toxicity of acetominophen and $\mathrm{N}$-acetyl-P-benzoquinone-imine in isolated hepatocytes is associated with thio depletion and increased cytosolic $\mathrm{Ca}^{2+}$. J. Biol. Chem. 260: 13035-13040, 1985.

Nakae, D., H. Yoshiji, K. Yamamoto, H. Maruyama, T. Kinugasa, Y. Takashima, A. Denda and Y. Konishi. Influence of timing of administration of liposome-encapsulated superoxide dismutase on its prevention of acetaminophen-induced liver cell necrosis in rats. Acta Pathol. Jpn. 40: $568-573,1990$.

Nelson, S.D. Molecular mechanisms of the hepatotoxicity caused by acetominophen. Sem. Liver Dis. 10: 267-278, 1990.

Ohkawa, H., N. Ohishi and K. Yagi. Assay for lipid peroxides in animal tissues by thiobarbituric acid reaction. Anal. Biochem. 95: 351-358, 1979.

Rees, K.R. and W.G. Spector. Reversible nature of liver cell damage due to carbon tetrachloride as demonstrate by the use of Phenergan. Nature (Lond.) 189: 821-829, 1961.

Rivera, E., F. Ekholm Pettersson, M. Inganas, S. Paulie and K.O. Gronvik. The Rb1 fraction of ginseng elicits a balanced Th1 and Th2 immune response. Vaccine 23: 5411-5419, 2005.

Rogers, L.K., B. Moorthy and C.V. Smith. Acetaminophen binds to mouse hepatic and renal DNA at human therapeutic doses. Chem. Res. Toxicol. 10: 470-476, 1997.

Sakaguchi, S., E. Tsutsumi and K. Yokota. Preventive effects of a traditional Chinese medicine (shosaiko-to) against oxygen toxicity and membrane damage during endotoxemia. Biol. Pharm. Bull. 16: 782-786, 1993.

Sinha, K.P. and A. Saran. A. Serum transaminase levels during the course of administration of carbon tetrachloride to rabbits. Ind. J. Med. Res. 60: 1378-1381, 1972.

Sugiyama, K., P. He, S. Wada and S. Saeki. Teas and other beverages suppress D-galactosamineinduced liver injury in rats. J. Nutr. 129: 1361-1367, 1999.

Taira, Z., K. Yabe, Y. Hamaguchi, K. Hirayama, M. Kishimoto, S. Ishida and Y. Ueda. Effects Shosaiko-to extract and its components, baicalin, baicalein, glycyrrhizin and glycyrrhetic acid, on pharmacokinetic behavior of salicylamide in carbon tetrachloride intoxicated rats. Food Chem. Toxicol. 42: 803-807, 2004.

Yamashiki, M., A. Nishimura, H. Suzuki, S. Sakaguchi and Y. Kosaka. Effects of the Japanese herbal medicine "sho-saiko-to" (TJ-9) on in vitro interleukin-10 production by peripheral blood mononuclear cells of patients with chronic hepatitis C. Hepatology 25: 1390-1397, 1997.

Zani, F., M.T. Cuzzoni, M. Daglia, S. Benvenuti, G. Vampa and P. Mazza. Inhibition of mutagenicity in Salmonella typhimurium by Glycyrrhiza glabra extract, glycyrrhizinic acid, 18 alpha- and 18 beta-glycyrrhetinic acids. Planta Med. 59: 502-507, 1993.

Zakowski, J.J. and A.L. Tappel. Purification and properties of rat liver mitochondrial glutathione peroxidase. J. Biol. Chem. 526: 65-76, 1978. 